\title{
Comportamiento innovador en individuos y grupos de trabajo: in- fluencia del capital psicológico colectivo, la direccionalidad estratégica y la facilidad para alcanzar acuerdos
}

\author{
Innovative behavior in individuals and work groups: Influence of collective \\ psychological capital, strategic directionality and the ability to reach agreements
}

\author{
Ronald Mora Esquivel, Martín Solís* \\ Instituto Tecnológico de Costa Rica \\ Recibido el 21 de marzo de 2018; aceptado el 1 de febrero de 2019 \\ Disponible en Internet el: 7 de febrero de 2019
}

\section{Resumen}

El presente estudio tiene como objetivo analizar el efecto mediador del capital psicológico y del acuerdo de grupo en la relación entre la direccionalidad estratégica con el comportamiento innovador tanto del individuo como del grupo de trabajo. Para ello, se aplicó un cuestionario a 231 sujetos que laboran en organizaciones estatales en Costa Rica. Se procesan los datos ejecutando dos modelos de medida y dos modelos estructurales mediante Smart-PLS 3.0. Los resultados obtenidos sugieren que la direccionalidad estratégica del grupo influye de manera directa sobre comportamiento innovador del grupo y de manera indirecta a través del capital psicológico colectivo y la facilidad de alcanzar acuerdos. Por otra parte, el capital psicológico colectivo influye de manera directa el comportamiento innovador del individuo. Los modelos analizados no han sido valorados previamente en la literatura, por lo cual, el presente estudio aporta nueva evidencia relativa a cómo potenciar el comportamiento innovador en grupos e individuos. Adicionalmente, los hallazgos han sido obtenidos de sujetos que laboran en instituciones públicas o de gobierno, lo cual resulta relevante considerando la escasa literatura y aporte empírico relacionado con el comportamiento innovador y emprendedor del empleado público.

Código JEL: M12, M19, O39

Palabras clave: Innovative behavior; Work groups; Individual; Psychological capital; Strategic directionality; Agreement

\footnotetext{
*Autor para correspondencia Correo electrónico: martin12cr@yahoo.com (M. Solís).

La revisión por pares es responsabilidad de la Universidad Nacional Autónoma de México. 


\begin{abstract}
The aim of this study is to examine the mediating role of Psychological Capital and Agreement between strategic directionality and innovative behavior of both individual and work team members. Data from 231 public sector organizations' employees in Costa Rica was collected. PLS-SEM technique was used, by Smart PLS 3.0 software, to estimate the parameters of two measurement models and two structural models proposed in this research. The study findings indicate that work group's perception of strategic directionality has a direct effect on work group innovative behavior, and revealed indirect effects mediated by collective Psychological Capital and Agreement. On the other hand, the empirical results provide support for the hypothesis that collective psychological capital increases the innovative behavior of individuals. The proposed models have not been previously tested in the literature, therefore the present study provides new evidence on how to enhance innovative behavior in work groups and individuals. As findings were obtained in Government and public sector organizations the study is an empirical contribution in the understanding of innovative and entrepreneurial behavior of public sector's employees considering the scarce literature on this topic.
\end{abstract}

JEL code: M12, M19, O39

Keywords: Innovative behavior; Work groups; Individual; Psychological capital; Strategic directionality; Agreement

\title{
Introducción
}

La innovación ha sido reconocida como un elemento notable para mantener la competitividad y mejorar el desempeño económico de las empresas (Dereli, 2015). Por su parte, se ha distinguido el papel que desempeña el recurso humano como fuente de ventaja competitiva; especialmente, si es tratado como un recurso de capital (Luthans y Youseef, 2004). De hecho, se ha afirmado que el comportamiento innovador de los colaboradores en las empresas -en el desarrollo, adopción e implementación de nuevas ideas para productos y métodos de trabajo- es considerado un activo que coadyuva en el éxito de una organización en entornos dinámicos (Yuan y Woodman, 2010).

Las ideas y las personas son parte de los elementos esenciales del proceso innovador; siendo las segundas quienes desarrollan las ideas, las conducen, reaccionan ante ellas y las modifican (Van de Ven, 1986; p. 592). Tanto, la etapa creativa -que se nutre de la generación de ideas-, como aquella que las concreta en nuevos o mejorados productos, servicios o procesos, se consideran fases interrelacionadas; mismas que son el resultado de los esfuerzos para lograr el desarrollo y la introducción de nuevas y mejores formas de hacer las cosas en el trabajo (Anderson, Potočnik y Zhou, 2014).

La literatura ha estudiado un tipo de comportamiento que ocurre cuando un individuo trata de hacer algo diferente, conocido como comportamiento innovador (Van Rijnsoever, Meeus 
y Donders, 2012). Este contempla todos aquellos esfuerzos intencionales que pretenden proporcionar resultados novedosos (Janssen, 2000). De esta manera se puede concebir como la suma de actividades físicas y cognitivas llevadas a cabo por los empleados en su contexto de trabajo, aisladamente o en un entorno social, para cumplir un conjunto de tareas necesarias que permitan el desarrollo de la innovación (Messman y Mulder, 2012.

El comportamiento innovador se ha estudiado desde la perspectiva del individuo (Krause, 2004; Janssen, 2000; Scott y Bruce, 1994) así como, en grupos de trabajo (Messman y Mulder, 2012; Post, 2012). Existe evidencia empírica que se ha enfocado en identificar factores que promueven este tipo de comportamiento (Scott, y Bruce, 1994; Yuan y Woodman, 2010; Romero y Martínez-Román, 2012). Hace más de una década, ha surgido una nueva corriente, denominada psicología organizativa positiva (Luthans y Youssef, 2004), que ha estado analizando el efecto de disposiciones motivacionales de los individuos (capital psicológico; Luthans, Avolio, Avey y Norman, 2007) en distintas áreas de su desempeño (e.g.: productividad del trabajador, Luthans, Avolio, Walumbwa y Li, 2005; creatividad, Sweetman, Luthans, Avey y Luthans, 2011; comportamiento innovador, Wojtczuk-Turek y Turek, 2015). No obstante, por tratarse de un constructo relativamente nuevo la investigación que ha intentado estudiar la conexión que existe entre capital psicológico y comportamiento innovador ha sido escasa (Schuckert, Kim, Paek y Lee, 2018).

El capital psicológico es considerado un activo intangible, valioso y que aporta a la competitividad de la empresa (Luthans y Youseef, 2004). Se afirma que los activos intangibles se han convertido en recursos clave para la creación de valor en la empresa; especialmente, si están alineados con la estrategia, con los objetivos de los procesos internos (Norton y Kaplan, 2004). En la revisión de literatura, llevada a cabo hasta ahora, no se logró identificar estudios empíricos que midan el alineamiento de objetivos en individuos y de grupos de trabajo como antecedente del capital psicológico. Lo que si se ha demostrado es el efecto directo que existe entre la claridad estratégica de equipos con el comportamiento innovador (Högl y Parboteeah, 2003; Hülsheger Anderson y Salgado, 2009; Caldwell y O'Reilly III, 2003; Janssen, Van de Vliert y West, 2004).

Ahora bien, existen dimensiones del capital psicológico (como la esperanza y autoeficacia) que están vinculados con motivaciones a las metas y objetivos (Luthans, Youssef y Avolio, 2007). En consecuencia, explorar el posible efecto mediador del capital psicológico entre la direccionalidad estratégica y el comportamiento innovador resulta oportuno para generar un aporte al conocimiento en esta nueva corriente. Lo anterior, es aún más notable por el hecho de que si bien se ha identificado estudios utilizando capital psicológico como variable mediadora, entre variables de liderazgo y el comportamiento innovador (Schuckert et al.,2018) y entre variables relativas a sistemas de recursos y el comportamiento innovador (Wojtczuk-Turek y Turek, 2015) no ha sido el caso en la vía que se está postulando en este 
estudio. No menos importante es valorar el efecto que ejerce la posibilidad de desarrollar acuerdos para crear una visión común (Revilla y Rodriguez, 2011) y su posterior efecto en el comportamiento innovador en los individuos y grupos de trabajo.

Por todo lo anterior, el presente estudio se traza como objetivo analizar, en la dinámica de grupos de trabajo, el efecto mediador del capital psicológico y del acuerdo de grupo en la relación entre la direccionalidad estratégica con el comportamiento innovador tanto del individuo como del grupo de trabajo. Para ello, se ha seguido un diseño de investigación cuantitativo, en el cual, se encuestó a 231 funcionarios de instituciones públicas en Costa Rica. Se busca contrastar un modelo que postula que la direccionalidad estratégica influye de manera directa en el comportamiento innovador del individuo y en el del grupo de trabajo; asimismo, que esta relación esta mediada por el capital psicológico y el desarrollo de acuerdos.

Se considera que el presente estudio tiene una doble contribución. En primer lugar, este entramado de relaciones no ha sido valorado previamente en la literatura, por lo cual, aporta nueva evidencia relativa al efecto de inductores motivacionales en el comportamiento innovador en individuos y en grupos de trabajo. En segundo lugar, el análisis se desarrolla con datos de colaboradores del sector público, lo cual, resulta relevante considerando la escasa literatura y aporte empírico relacionado con el comportamiento innovador y emprendedor del empleado público (Shoham, Vigoda $\square$ Gadot, Ruvio y Schwabsky, 2012), lo mismo que, de posibles inductores e inhibidores de la innovación en este sector (Potts y Kastelle, 2010).

El artículo se estructura de la siguiente forma. En la siguiente sección se expone los antecedentes e hipótesis de investigación. Posteriormente se presenta la muestra sobre la cual se obtuvieron los datos, las variables latentes reflectivas con sus respectivas escalas de medida y el procedimiento utilizado. Seguidamente, se muestran los resultados del estudio y finalmente, se discuten los resultados, así como, las limitaciones y futuras líneas de investigación.

\section{Antecedentes e Hipótesis de Investigación}

\section{Dirección estratégica y comportamiento innovador}

El comportamiento innovador ha sido conceptuado como una forma de comportamiento que ocurre cuando un individuo intenta algo nuevo (Van Rijnsoever et al., 2012); o dirige esfuerzos que pretenden proporcionar resultados novedosos (Janssen, 2000). Por su parte, Kleysen y Street (2001, p. 285) lo definen como todas aquellas acciones que acometen los individuos orientadas hacia la generación, introducción o aplicación de una novedad que resulte útil a cualquier nivel de la organización. Es esta última definición por la que se decanta el presente estudio.

En el ámbito estratégico, el desarrollo e implementación de un conjunto de objetivos y políticas funcionales, coherentes a lo interno y que definan colectivamente su posición en el 
mercado, ha sido reconocido como una de las condiciones que determinarían una posición de desempeño superior o sostenible (Porter, 1991). Asimismo, un buen entendimiento de una estrategia global, en toda la organización, permitiría filtrar diversas acciones y, a los individuos, diseñar sus propias formas de contribuir a la estrategia (Porter, 1991).

Desde la teoría del establecimiento de metas se ha postulado que la meta afecta la acción (Locke, 1996, Locke y Latham, 2002). A lo largo de más de cinco décadas de estudios experimentales y de campo, la literatura al respecto ha aportado evidencia que reconoce el vínculo entre el establecimiento de metas y el desempeño y, por ende, el valor práctico de esta perspectiva teórica en el ámbito organizativo (Latham y Yukl, 1975; O’Leary-Kelly, Martocchio y Frink, 1994; Locke y Latham, 2002).

Según esta teoría, dirigir la atención hacia actividades relevantes, la función dinamizadora que permite movilizar el esfuerzo, la posibilidad de aumentar la persistencia y de motivar el desarrollo de la estrategia son mecanismos principales por los cuales las metas afectan el desempeño (Locke, Shaw, Saari, y Latham, 1981; Locke y Latham, 2002). Finalmente, estudios previos han encontrado que la dificultad de la meta y su grado de especificidad guardan relación directa con el desempeño. En la primera, se parte de la premisa que el individuo dispone de las habilidades y conocimiento para acometerlas; mientras que en la segunda, la reducción en la ambigüedad, en lo relativo a lo que espera obtener, permite reducir la variación en el desempeño (Locke y Latham, 2002)

En línea con Anderson y West (1996) el grado en que los objetivos y la visión del equipo se defina con claridad, se comparta, se considere alcanzable y se valore permite delimitar la orientación a los objetivos. Por su parte, Pearce y Ensley (2004) definen visión compartida como un modelo mental común relativo al estado futuro del equipo o de sus tareas, el cual, prepara las bases para el funcionamiento dentro del equipo. Tomando como referencia las consideraciones de estos autores y la perspectiva teórica del establecimiento de metas, el presente estudio concibe la direccionalidad estratégica como la claridad de propósito que brinda significado al individuo y a los grupos de trabajo de lo que se quiere lograr, con una visión y metas claramente definidas, una estrategia y planificación para alcanzarlas.

En el ámbito empírico existe evidencia que permite apoyar el vínculo entre la direccionalidad estratégica y el comportamiento innovador. En su meta-análisis, Hülsheger et al. (2009) destacan la visión como uno de los procesos de equipo de trabajo que conducen, en forma sustancial y significativa, a la innovación. Conforme al estudio longitudinal de Pearce y Ensley (2004) se acentúa el papel central que juega la visión compartida en los procesos de innovación. Además, hacerla clara resulta ser medular para intensificar los esfuerzos innovadores en los equipos. Mas aún, encontraron un efecto recursivo en el sentido que los resultados innovadores reforzaban, a su vez, la visión de grupo (Pearce y Ensley, 2004, p.274). Por su parte, el estudio de Cardinal (2001), referente a equipos de trabajo en la industria farmacéu- 
tica, mostró la existencia de un efecto directo y positivo de la especificación de metas no sólo con las innovaciones incrementales sino también con el desarrollo de nuevos productos. La primera concebida como el punto en que las metas son explicitas, claramente definidas y proveen criterios sin ambigüedad para elegir entre alternativas. Todavía cabe señalar que Anderson y West (1998) afirman que es más propenso desarrollar nuevos métodos de trabajo apropiados para los objetivos, en grupos de trabajo que cuentan con objetivos claramente definidos, por motivo que sus esfuerzos están enfocados y tienen dirección.

En consonancia con lo anterior, Högl y Parboteeah (2003) evidenciaron que el establecimiento de metas correlacionó positivamente con medidas de efectividad en equipos desarrolladores de software. Sugieren que este resultado se debe a que este tipo de medidas resultan ser más realistas que las medidas de eficiencia en proyectos innovadores. Igualmente, la literatura ha evidenciado que aquellos colaboradores que cuentan con una claridad de lo que se espera de ellos son más propensos que dirijan sus esfuerzos hacia el cumplimiento de objetivos o estándares esperados para el trabajo. Esto ha sido reconocido no solo como una característica en equipos exitosos (Franz, 2004) sino también como un inductor de la implementación de innovaciones (Holleman, Poot, Mintjes-de Groot y van Achterbergü, 2009). Por su parte, Gilson y Süalley (2004) hallaron que equipos con niveles más altos de participación en enfoques creativos para la solución de problemas mostraron mayor grado de compromiso y sentido de meta compartida. Además de los estudios citados, otras investigaciones han señalado la relevancia de metas claras y compartidas para desarrollar innovación (Caldwell y O’Reilly III, 2003; Janssen et al., 2004), así como, una estrategia y planificación orientada hacia el logro de esos objetivos ( Koch y Hauknes, 2005). Todo lo anterior permite postular, como hipótesis, que:

H1a: La direccionalidad estratégica ejerce un efecto positivo en el comportamiento innovador del grupo.

H1b: La direccionalidad estratégica ejerce un efecto positivo en el comportamiento innovador del individuo.

\section{Dirección estratégica y capital psicológico}

Un nuevo movimiento, denominado psicología positiva, ha puesto atención al plano productivo y valioso de la vida de las personas, así como materializar su potencial humano. Hace más de década y media, dentro de este movimiento, ha brotado una propuesta de corriente llamada conducta organizativa positiva (Luthans y Youssef, 2004). Esta postula que las potencialidades del recurso humano se pueden orientar y que existen capacidades psicológicas medibles, desarrollables y gestionables para mejorar el rendimiento en el lugar de trabajo. 
Como resultado, surge un nuevo constructo que representa disposiciones motivacionales de los individuos, los cuales emanan de constructos de la psicología positiva, como lo son la eficacia, el optimismo, la esperanza y la resilencia (Luthans et al., 2007). El mismo lo denominan capital psicológico, reconocido como recurso valioso para las organizaciones (Luthans y Youssef, 2004).

Luthans, Youssef y Avolio (2007) lo definen como un estado de desarrollo psicológico positivo del individuo que se caracteriza por cuatro dimensiones o rasgos; a saber: a) una autoconfianza para asumir y emprender esfuerzos necesarios para asumir con éxito tareas demandantes; b) atribuirse una actitud positiva respecto a obtener éxito hoy y en el futuro; c) una capacidad para perseverar en los objetivos y, cuando sea necesario, la confianza en reorientar los caminos hacia los objetivos para tener éxito; y d) la suficiencia para levantarse ante los problemas y adversidades para alcanzar el éxito (p. 3). Si bien, ha sido un constructo analizado, principalmente, en el ámbito del individuo, como un estado psicológico donde prevalece la actitud positiva; también ha sido posible analizarlo en el ámbito de grupos de trabajo (Walumbwa, Luthans, Avey y Oke, 2011, Luthans et al., 2007).

Si bien se ha afirmado que la orientación a metas puede inducir el desempeño en colaboradores y grupos de trabajo, la literatura ha reconocido que la primera no necesariamente es condición suficiente; por tanto, otras variables estarían interviniendo en esta relación (Locke y Latham, 2002; Camelo-Ordaz, Fernández-Alles y Valle-Cabrera, 2008; Bart, Bontis y Taggar, 2001; Boswell, 2006). A continuación, se presentan argumentaciones relativas al posible vínculo entre la dirección estratégica y dimensiones del capital psicológico

Locke y Latham (2002) presentan estudios que permiten afirmar que el grado de compromiso de las personas hacia la meta actuaría dentro de esta relación. Esta variable estaría asociada positivamente a tres impulsores: a) la dificultad de la meta, al demandar en las personas un mayor esfuerzo; b) el grado de creencia de las personas que es posible alcanzar la meta; mismo que está en línea con estudios citados por Pearce y Ensley (2004, p.262263) que han mostrado que el comprender y dar reconocimiento a los objetivos afecta positivamente la confianza y autoeficacia para lograr las metas; c) la influencia de líderes que pueden comunicar e inspirar la visión, actuando como apoyo a otros. Por su parte, Luthans, Avey y Patera (2008) afirman que metas y objetivos claros podrían reducir las expectativas negativas en los colaboradores. Igualmente, Kirkpatrick y Locke (citados por Levin, 2000, p. 92) han demostrado que la visión ejerce un impacto positivo tanto en las actitudes como en el desempeño de los colaboradores.

En su meta-análisis Gully, Incalcaterra, Joshi y Beaubien (2002) refieren a Guzzo y Shea quienes han configurado el constructo denominado potencia grupal, cuya evidencia empírica constata estar relacionado con el desempeño. El mismo, alude a las creencias, generalizadas, de las capacidades que percibe el equipo en todas las tareas y contextos "(esto es, nuestro 
equipo tendrá éxito sin importar cuál sea la tarea)”. En su estudio Pearce y Ensley (2004) demuestran que la visión compartida del grupo incide positivamente en la potencia grupal. Además, no solo corrobora que dicha visión refuerza las creencias positivas que tienen los miembros de sus habilidades para asumir con éxito sus tareas, sino también, que esto último, fortifica la visión compartida de grupo, en forma recursiva. Todos estos antecedentes permiten conjeturar que:

H2a: La direccionalidad estratégica ejerce un efecto positivo en el capital psicológico del grupo. $\mathrm{H} 2 \mathrm{~b}$ : La direccionalidad estratégica ejerce un efecto positivo en el capital psicológico del individuo.

\section{Capital psicológico y comportamiento innovador}

La dimensión optimismo del capital psicológico puede ejercer influencia sobre el comportamiento innovador, tanto de colectivos como de individuos, al facilitar la apertura al cambio, la toma de riesgos, el establecimiento de metas y el cumplimiento de objetivos (Green, Medlin y Whitten, 2004). Según Shani y Divyapriya (2011) una mentalidad positiva es más creativa porque genera confianza en sí mismo y considera los errores como una oportunidad de aprendizaje. Los hallazgos de Avey, Wernsing y Luthans (2008) demuestran que el capital psicológico influye en las actitudes y comportamientos de los individuos que potencian el cambio organizacional, en parte porque las prácticas y emociones positivas podrían fomentar la intención de generar cambios en la efectividad de una organización (Cameron, Mora, Leutscher y Calarco, 2011).

En otro orden, la literatura ha destacado, como característica del individuo que contribuye a la innovación, cierto grado de fuerza interna que mantiene a la persona en marcha inclusive en situaciones en que los desafíos se superan con éxito (Parzefall, Seeck y Leppänen, 2008). Se ha descrito como una cuestión de tensión positiva, perseverancia y deseo de superación. En este sentido, es probable que algunas personas posean un mayor impulso y una necesidad de logro que las animen a permanecer intrínsecamente motivadas (Parzefall et al., 2008).

En lo relativo al rasgo esperanza del capital psicológico, estudios de Rego, Marques y Cunha (2012) han encontrado que esta capacidad de orientación a las metas y empeño en redireccionar esfuerzos hacia el logro de objetivos nutre la creatividad. Por otro lado, en lo referente al rasgo auto-eficacia, se ha encontrado que la eficacia colectiva ayuda a la motivación del grupo porque los miembros confían más en los demás para cumplir las tareas (Bandura, 1997). Cuando enfrentan obstáculos, los equipos con alta eficacia colectiva persisten en tratar de resolver los problemas (Bandura, 1997). 
Entre la escasa evidencia empírica que ha demostrado el vínculo entre capital psicológico y el comportamiento innovador, los estudios de Sweetman et al. (2011) y Zubair y Kamal (2015), basados en regresiones, mostraron una relación positiva entre capital psicológico y la creatividad de los colaboradores. Los trabajos de Jafri (2012) y de Abbas y Raja (2015) encontraron una relación significativa entre capital psicológico y conducta innovadora, en modelo de regresión; empero, explicando entre 4\% y 5\% de la varianza de la variable dependiente. Por su parte, Ratnaningsih, Prasetyo y Prihatsanti (2016) encontraron una correlación significativa $(r=.519, \mathrm{p}<.001)$ entre estas dos variables. Finalmente, los estudios de Wojtczuk-Turek y Turek (2015), lo mismo que Schuckert et al. (2018), empleando modelos de ecuaciones estructurales, han mostrado efecto significativo del capital psicológico en el comportamiento innovador $(\beta=.73, \mathrm{p}<.01 ; \beta=.275, \mathrm{t}=4.436, \mathrm{p}<.001$, respectivamente) explicando, por consiguiente, $53 \%$ y $7,5 \%$ de la varianza del comportamiento innovador, respectivamente. Por todo lo descrito con anterioridad, se plantean las siguientes hipótesis:

H3a: El capital psicológico, a nivel de grupo, influye de manera positiva el comportamiento innovador del grupo.

$\mathrm{H} 3$ b: El capital psicológico, en el individuo, influye de manera positiva el comportamiento innovador del individuo.

\section{Dirección estratégica y acuerdo}

Desde la óptica de una gestión estratégica del recurso humano, en el momento que los colaboradores perciben claridad y consistencia entre el mensaje de los tomadores de decisión estratégica con las prácticas de recursos humanos es más probable que se facilite el consenso o acuerdo dentro de los colaboradores en la organización (Bowen y Ostroff, 2004). El consenso se puede concebir como la existencia de acuerdo, entre los colaboradores, en su perspectiva de la relación entre evento-efecto; en lo relativo a los objetivos de influencia previstos por el sistema de gestión del recurso humano (p.212). En particular, Holland, Gaston y Gomes (2000) afirman que uno de los elementos clave para que un grupo de trabajo asuma una responsabilidad, en forma colectiva, que solvente un conjunto diverso de demandas es "ser muy cohesivo con metas claras" (p.248). Tomando como referencia a Fey y Denison (2003), Denison, Janovics y Young (2006), en este estudio se conceptúa acuerdo como la capacidad de líderes y seguidores de llegar a acuerdos (incluso con diversos puntos de vista) y de reconciliar las diferencias cuando ocurren.

Acorde con la idea propuesta por Schneider (1987) las metas mantienen el interés de las personas por permanecer en una organización y postula que, por medio de estas, es que los individuos interactúan. El grado de acuerdo en las metas entre individuos y superiores -perspectiva 
denominada congruencia en la meta- ha sido probado que se relaciona positivamente con la satisfacción en el trabajo y el compromiso organizativo (Vancouver, Millsap y Peters, 1994). De igual modo, en el ámbito de pequeñas y medianas empresas, Jing, Avery y Bergsteiner (2014) han comprobado que una visión debe ser comunicada y compartida entre líderes y seguidores, para obtener los beneficios de un mayor desempeño. Lo anterior ocurre por motivo que una visión compartida influye en las actitudes hacia el trabajo, en especial, en la satisfacción al trabajo.

En consonancia con lo anterior, Boswell (2006) postula la importancia del alineamiento de visión en los colaboradores como factor que incide en actitudes respecto al trabajo y, por ende, en medidas de su desempeño. Esta se conceptualiza como la comprensión que tiene el colaborador de los objetivos estratégicos y, en especial, de cómo contribuir a su logro. En su estudio demuestra que este último aspecto es quizás el más importante para mantener adheridos a los colaboradores a la organización, ya que les brinda un mayor sentido de pertenencia, de congruencia en la meta y satisfacción con el trabajo; más que el simple hecho de conocer los objetivos estratégicos.

Por su parte, la diversidad de funciones y tareas que conlleva el trabajo de un equipo, en ausencia de una visión común entre sus miembros, puede llevar a conflictos y desacuerdos en el contenido de las tareas y las acciones necesarias para acometerlas (Revilla y Rodriguez, 2011). El compartir propósitos y objetivos comunes coadyuvaría a desarrollar un sentido de responsabilidad mutua lo que conllevaría, a su vez, a desplegar confianza y compromiso en el grupo, ejerciendo un efecto positivo en su desempeño (Katzenbach y Smith, 1993). Además, cuando los colaboradores comprenden el qué hacer y el qué se espera de ellos, experimentan una mayor satisfacción en el trabajo ya que les permite contar con un sentido de propósito, de enfoque y de dirección en sus actividades diarias (Bart et al., 2001). Todo lo anterior, lleva a postular las siguientes hipótesis:

H4a: La dirección estratégica influye positivamente el nivel de acuerdo del grupo.

H4b: La dirección estratégica influye positivamente el nivel de acuerdo del individuo.

\section{Acuerdo y comportamiento innovador}

Anderson y West (1998) coinciden que el apoyo a la innovación puede variar entre los grupos de trabajo, en la medida en que ésta se articula y promulga. Estos autores son del criterio que, en los grupos, no es suficiente el apoyo articulado -basado en declaraciones de política, documentos de personal o por transmisión boca a boca; adicionalmente, es necesario el apoyo por aprobación para la innovación grupal.

En su revisión de literatura Kozlowski e Ilgen (2006) afirman que la cohesión del grupo está vinculada con su desempeño. Además, que esta relación se fortalece a medida que las 
demandas de flujo de trabajo del grupo aumentan la interdependencia y requieren una mayor coordinación de información y esfuerzo. Estudios empíricos han aportado evidencia de la contribución del trabajo en equipo, la comunicación y la coordinación entre sujetos como antecedentes para detonar la creatividad (Luke, Verreynne y Kearins, 2010; Shalley y Gilson, 2004). Por su parte, otros trabajos han subrayado que legitimar una idea innovadora y concretar su apoyo en la etapa de implementación son necesarios para impulsar la innovación (Hogan y Coote, 2014; Jamrog, Vickers y Bear, 2006; Jassawalla y Sashitta, 2002).

La facilidad para alcanzar acuerdos coadyuva al desarrollo de un sentido de propósito conjunto y significado común, impulsando a los individuos hacia la mejora y la innovación (Shalley y Gilson, 2004). Todo lo anterior conlleva a conjeturar que entre más facilidad exista para alcanzar acuerdos, es más factible lograr ese involucramiento y apoyo de diferentes individuos para trabajar colectivamente, coordinar y comunicarse adecuadamente en el proceso de implementación de una idea innovadora. En consecuencia, se postulan como hipótesis, lo siguiente:

H5a: El grado de acuerdo influye de manera positiva el comportamiento innovador del grupo. H5b: El grado de acuerdo influye de manera positiva el comportamiento innovador del individuo.

En síntesis, la Figura 1 resume los dos modelos teóricos propuestos a ser contrastados empíricamente en esta investigación.
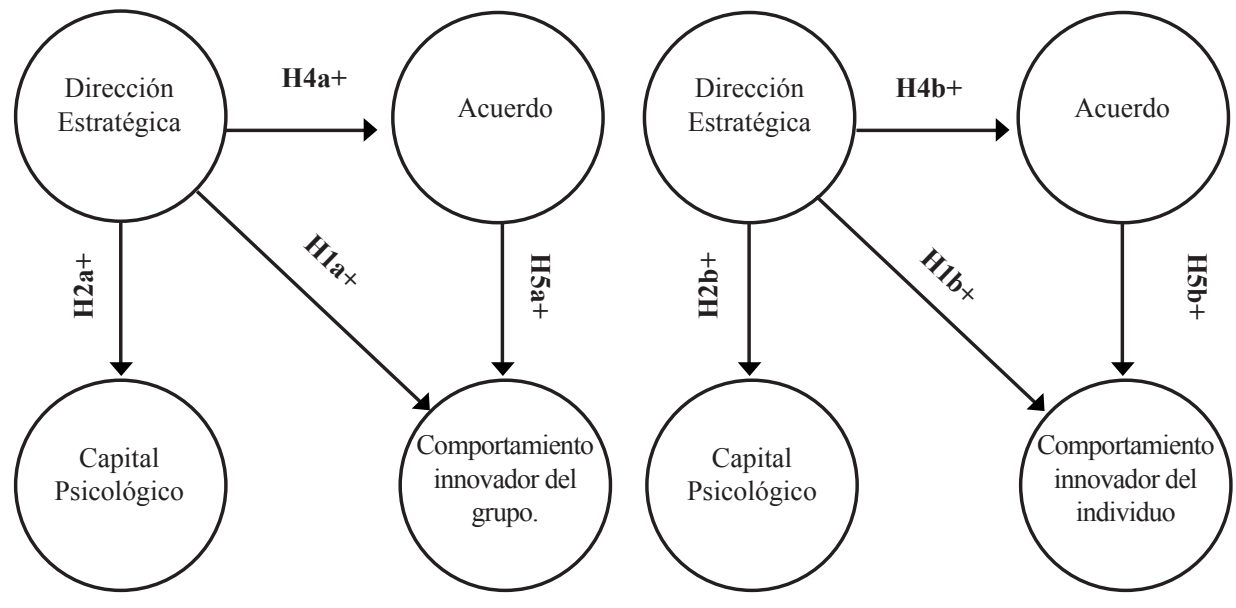

Figura 1.Modelos teóricos de inductores del comportamiento innovador del grupo y del individuo Fuente: Elaboración propia 


\section{Metodología}

Muestra

La unidad de estudio la conformó el funcionario público que está cursando postgrado, en universidades públicas de Costa Rica, en tópicos relativos a la Gestión Pública. Se elige esta unidad, porque facilita la ubicación de funcionarios públicos para la aplicación del instrumento de medición. Para obtener la muestra se recurrió a nueve grupos de Maestrías en tres universidades estatales costarricenses. Se visitó los grupos para autoadministrar un cuestionario a los estudiantes presentes, siempre y cuando cumplieran como condición estar laborando en una institución pública. En total se logró obtener respuesta de 231 funcionarios públicos. En la Tabla 1 se presentan las características de los entrevistados.

Como se puede apreciar en la Tabla 1, poco más de la mitad de los consultados fueron mujeres, un tercio de los encuestados ocupan cargos de jefatura y la mayoría trabajan en el sector de salud. En el ámbito institucional, la mayor parte son semi-autónomas, esto es, instituciones públicas que tienen autonomía del Gobierno Central para su gestión; por ejemplo: Universidades Públicas, Hospitales y otros Centros de Salud, Instituciones Públicas que brindan servicios de seguros, electricidad, abastecimiento de agua, Municipalidades, entre otras. Por otro lado, una pequeña parte de esas instituciones (20\%) son del Gobierno Central, por ejemplo: Ministerio de Obras Públicas, Ministerio de Salud, Ministerio de la Presidencia, entre otros. Cabe señalar que a lo sumo un $15 \%$ de los sujetos pertenecían a una misma institución. La edad promedio de los entrevistados es de 37 años ( $\mathrm{DE}=8.6)$; el tiempo medio de laborar en la institución es 11 años y el tiempo medio de trabajar en el departamento donde se encuentran actualmente es de 6 años.

Tabla 1

Estadísticas descriptivas de los entrevistados, $\mathrm{N}=231$

\begin{tabular}{llll}
\hline Variables categóricas & Proporción & \\
\hline Género & & - & - \\
Mujeres & $58.0 \%$ & - & \\
Hombre & $42.0 \%$ & - & - \\
Posee jefafura & & - & - \\
Sí & $33.0 \%$ & & \\
No & $77.0 \%$ & -
\end{tabular}




\begin{tabular}{llll} 
Infraestructura & $6.7 \%$ & - & - \\
Educación & $6.7 \%$ & - & - \\
Otras & $25.6 \%$ & - & Máximo \\
\hline Variables cuantitativas & Media & 22.0 & 70.0 \\
\hline Edad & 37.4 & 0.17 & 27.2 \\
Años en la unidad & 6.0 & 0.42 & 42.0 \\
Años en la institución & 10.9 & & \\
\hline
\end{tabular}

Fuente: Elaboración propia

\section{Definición de variables latentes}

A continuación, se describe brevemente como fueron medidos cada uno de los constructos del estudio. El detalle de los ítems utilizados para medirlos se presenta en el Apéndice A.

\section{Comportamiento innovador del grupo}

Para medirlo se tomaron cinco ítems de la escala propuesta por De Jong y Den Hartog, (2010) (e.g.: En mi departamento se introducen sistemáticamente nuevas o mejoradas prácticas de trabajo). Sin embargo, dos de ellos recibieron cambios considerables en la fase de la prueba piloto como resultado de la aplicación de la entrevista cognitiva (procedimiento que se explicará en el siguiente apartado). Los ítems fueron valorados mediante una escala de respuesta tipo Likert de 5 puntos, desde $1=$ nada frecuente hasta $5=$ muy frecuente.

\section{Comportamiento innovador del individuo}

Se tomaron los ítems de la escala de Scott y Bruce (1994) (e.g.: Busca nuevos métodos de trabajo o ideas de nuevos servicios), los cuales, son evaluados por medio de una escala de respuesta Likert de 5 puntos, donde $1=$ nada frecuente y $5=$ muy frecuente.

\section{Direccionalidad estratégica}

Para medir este constructo se utilizó 3 ítems, de Denison et al. (2006) (e.g.: En mi departamento comparamos continuamente nuestro progreso con los objetivos fijados), empero, se elaboró uno adicional por parte de los investigadores (Mi departamento se caracteriza por una planificación constante del trabajo que hacemos). Los cuatro ítems son evaluados con una escala de respuesta Likert de 6 puntos, donde 6=muy alta o total semejanza con la unidad de 
trabajo, $5=$ =alta , 4=media alta, 3=media baja, 2=baja, 1=muy baja 0 ninguna semejanza con la unidad de trabajo.

\section{Capital psicológico colectivo}

Fue medido con 3 ítems de la escala propuesta por Walumbwa et al. (2011) (e.g.: En mi departamento hay optimismo de los resultados que obtendremos a futuro con el trabajo que realizamos) y un ítem propio que se agregó a la escala (e.g.: cuando enfrentamos dificultades propias de nuestras tareas somos muy insistentes en sacar adelante lo que nos proponemos). Para su valoración se utilizó el mismo tipo de escala de respuesta Likert de 6 puntos mencionada en el párrafo anterior.

\section{Acuerdo}

Fue medido con dos ítems de Denison et al. (2006) (e.g.: Cuando existe desacuerdos trabajamos intensamente para encontrar soluciones donde todos ganen) y otro ítem adicional, elaborado por el equipo investigador (Generalmente las personas tienen disposición de ceder su interés particular para lograr acuerdos en el grupo). Para su valoración se utilizó el mismo tipo de escala de respuesta Likert de 6 puntos mencionada en el párrafo tras anterior.

\section{Variable de marcado para medir riesgo de sesgo de varianza común}

Se debe anotar que las variables latentes dependientes del estudio (comportamiento innovador del grupo y comportamiento innovador del individuo) al ser medidas con las respuestas de los mismos individuos que evalúan los constructos direccionalidad estratégica, capital psicológico y acuerdo, puede generar el sesgo de varianza común cuando se analizan la relaciones entre las variables dependientes e independientes. Este sesgo corresponde con la varianza espuria atribuible al método de medición en lugar de la varianza atribuible a los constructos que representan las medidas (Podsakoff, MacKenzie y Podsakoff, 2012). Una forma de controlar el efecto de este sesgo es incorporando una variable de marcado (rasgo que no esté relacionado con las medidas de interés) en el modelo estructural. Por lo anterior, se incorporó una variable latente medida con tres ítems que evalúan el grado de ruido existente en el espacio de trabajo del entrevistado. Estos tres ítems fueron: i) el ambiente de trabajo en mi unidad es poco ruidoso, i) es poco común que se experimenten distracciones auditivas en el espacio de trabajo de mi unidad, iii) en mi unidad resulta fácil concentrarse para realizar las tareas. 


\section{Procedimientos}

En una primera fase se procedió a traducir, al idioma español, los ítems de las escalas señaladas en el apartado anterior. Para ello, se utilizó la técnica de doble traducción (back-translation) sugerida por Brislin (1986) y en especial, el método seguido por Beaton y Guillemin (2000). Se procesaron los datos siguiendo el formato propuesto por Vargas-Halabí, Mora-Esquivel y Siles (2017). Esto permitió completar el cuestionario, el cual, en una segunda fase fue sometido a un pretest (Colton y Covert, 2007). Para ello, se recurrió a la Entrevista Cognitiva, considerada como una de las técnicas importantes para identificar y corregir problemas en preguntas de un cuestionario (Beatty y Willis, 2007, p. 287). Se entrevistó a 6 sujetos. Se utilizó el protocolo diseñado por Smith-Castro y Molina (2011) y el formato propuesto en Vargas-Halabí y Mora-Esquivel (2017). Se identificaron y corrigieron los principales problemas de comprensión de ítems. Seguidamente, se procedió a recolectar los datos y continuar la siguiente fase del análisis de los datos.

En la tercera fase se evaluaron los dos modelos de medida y los dos modelos estructurales propuestos en la Figura 1. Para el procesamiento de los datos se empleó el software PLS-SEM 3.0 (Ringle, Wende y Becker, 2015), siguiendo el protocolo de dos etapas recomendado por Hair Jr., Hult, Ringle y Sarstedt (2017), con los niveles aceptables de parámetros sugeridos en cada una de ellas. En la primera etapa, correspondió evaluar los dos modelos de medida para cada una de las variables latentes reflectivas, segun el siguiente procedimiento: a) verificar el umbral de las cargas que miden la relación entre variables latentes reflectivas y sus indicadores (outerloadings, por su término en inglés), b) valorarar la consistencia interna de los modelos de medida, mediante el Alfa de Cronbach y la Confiabilidad Compuesta (CR, por su sigla en inglés), c) valorar el promedio de varianza extractada (AVE, por su sigla en inglés), para verificar validez convergente, d) analizar las correlaciones entre cargas cruzadas de variables latentes, el criterio Fornell-Larcker, la razón múltiple rasgo-unirasgo (HTMT, por su sigla en inglés) de correlaciones entre variables latentes y los intervalos de confianza de la razón HTMT (mediante procedimiento boostraping), para obtener evidencias de validez discriminante.

Comprobada la confiabilidad y la validez de los modelos de medida, se procedió, en una segunda etapa, a evaluar la capacidad predictiva de ambos modelos estructurales propuestos por medio de criterios heurísticos (Hair Jr., et al., 2017). Para ello, se verificó: a) la ausencia de niveles críticos de colinealidad entre cada grupo de variables predictoras según criterios de Tolerancia y el Factor de Inflación de la Varianza (VIF, por su sigla en inglés); b) la significancia estadística de los coeficientes path del modelo; c) los niveles de coeficientes de determinación (R2); d) la valoración del tamaño del efecto (f2) para medir el impacto en el cambio en R2 en variable latente endógena al omitir constructos exógenos. Finalmente, se 
realizó un test de significancia de los efectos de mediación propuestos en los modelos estructurales mediante la alternativa del boostraping, que permite el software Smart-PLS 3.0, para los efectos indirectos específicos y los efectos totales.

Para controlar el sesgo de varianza común en un modelo PLS-SEM, se siguieron las pautas de Rönkkö y Ylitalo (2011). De acuerdo con estos autores el constructo que evalúa la variable de marcado -en este estudio, grado de ruido-, debe relacionarse de manera directa con las todas las variables endógenas del modelo estructural.

\section{Resultados}

Esta sección presenta los resultados obtenidos de los modelos de investigación utilizando la técnica PLS-SEM, en dos etapas. Inicialmente, se mostrarán los indicadores esenciales de los dos modelos de medida y, posteriormente, los resultados de los dos modelos estructurales.

\section{Modelos de medida}

En la Tabla 2 se puede observar que cada variable latente reflectivo mostró ítems con cargas superiores a 7 (Chin, 1998; Henseler, Ringle y Sinkovics, 2009; véase las columnas 2 y 6 , respectivamente, en Tabla 2). Los valores de los Alfa de Cronbach de las variables latentes se ubicaron entre .84 y .93 , considerados muy buenos (DeVellis, 2012, p.109). Por su parte, los correspondientes a Confiabilidad Compuesta se ubicaron entre .90 y .95 que superan el umbral mínimo deseable de .7 (Hair Jr., et al., 2017, p.111). Por consiguiente, las medidas de los constructos reflectivos muestran niveles satisfactorios de consistencia interna (Véase las columnas 4 y 8 de la Tabla 2). Por su parte, el AVE de cada variable latente supera el umbral de .50 (Hair Jr., et al., 2017, p.115), mostrando evidencias de validez convergente y, por ende, que los constructos explican más de la mitad de la varianza de sus ítems.

Tabla 2

Valores de criterios para evaluar cada uno de los modelos de medida

Modelos de medida relativos a constructos Modelos de medida relativos a constructos reflectivos DEs, CPs, Ac y CIG reflectivos DEs, CPs, Ac y CII

\begin{tabular}{lllllllll}
\hline Item & Cargaa & AVE & CR & Alpha & Cargaa & AVE & CR & Alpha \\
\hline CIG & & .78 & .95 & .93 & & & & \\
CIG_1b & .84 & & & & - & - & - & - \\
CIG_2 & .86 & & & & - & & & \\
CIG_3 & .89 & & & & - & & \\
CIG_4 & .89 & & & & - & &
\end{tabular}




\begin{tabular}{|c|c|c|c|c|c|c|c|c|}
\hline CIG_5 & .92 & & & & - & & & \\
\hline CII & & & & & & .65 & .94 & .92 \\
\hline CII_1 & - & - & - & - & .81 & & & \\
\hline CII_2 & - & & & & .84 & & & \\
\hline CII_3 & - & & & & .82 & & & \\
\hline CII_4 & - & & & & .79 & & & \\
\hline CII_5 & - & & & & .86 & & & \\
\hline CII_6 & - & & & & .79 & & & \\
\hline CII_7 & - & & & & .82 & & & \\
\hline CII_8 & - & & & & .72 & & & \\
\hline DEs & & .71 & .91 & .86 & & .71 & .91 & .86 \\
\hline DEs_1 & .80 & & & & .80 & & & \\
\hline DEs_2 & .89 & & & & .89 & & & \\
\hline DEs_3 & .85 & & & & .85 & & & \\
\hline DEs_4 & .83 & & & & .83 & & & \\
\hline Ac & & .75 & .90 & .84 & & .75 & .90 & .84 \\
\hline Ac_1 & .89 & & & & .89 & & & \\
\hline Ac _2 & .86 & & & & .86 & & & \\
\hline Ac_3 & .86 & & & & .86 & & & \\
\hline CPs & & .73 & .91 & .87 & & .73 & .91 & .87 \\
\hline CPs_1 & .91 & & & & .91 & & & \\
\hline CPs_2 & .77 & & & & .78 & & & \\
\hline CPs_3 & .88 & & & & .88 & & & \\
\hline CPs_4 & .84 & & & & .84 & & & \\
\hline
\end{tabular}

Nota: aCorresponde a loa outerloadings; bSe asignan números a cada indicador del constructo reflectivo correspondiente. $\mathrm{DEs}=$ Dirección estratégica, $\mathrm{CPs}=$ Capital Psicológico, Ac=Acuerdo, $\mathrm{CIG}=\mathrm{Comportamiento}$ innovador del grupo, $\mathrm{CII}=$ Comportamiento innovador del individuo, $\mathrm{AVE}=$ Promedio de Varianza Extractada; $\mathrm{CR}=$ Confiabilidad Compuesta, Alpha $=$ Alpha de Cronbach

Fuente: Elaboración propia

El criterio de Fornell y Larcker, para evaluar la validez discriminante, se cumple en ambos modelos de medida. Como lo muestra la Tabla 3, la raíz del promedio de la varianza extractada de cada variable latente (en la diagonal) es mayor que su correlación con las otras variables latentes. Esto es un indicativo que cada constructo comparte más varianza con sus indicadores asociados que con los de otros constructos. 
Tabla 3

Criterio Fornell-Larcker para validez discriminante

\begin{tabular}{lccc}
\hline Variables latentes & CIG - CIIa & DEs & Ac \\
\hline CIG- CII & $.88-.81$ & & \\
DEs & $.65-.23$ & .84 & .87 \\
Ac & $.68-.31$ & .73 & .82 \\
CPs & $.72-.32$ & .79 & .85 \\
Nota: aValores a la izquierda del guion corresponden al CIG, mientras que los de la derecha al CII. En negrita se \\
encuentra la raíz de la varianza extractada, los valores restantes son las correlaciones entre las variables latentes. \\
DEs=Dirección estratégica, CPs=Capital Psicológico, Ac=Acuerdo, CIG=Comportamiento innovador del grupo, \\
CII=Comportamiento innovador del individuo.
\end{tabular}

Fuente: Elaboración propia

La funcionalidad del criterio Fornell-Larcker puede presentar problemas (Hair Jr., et al., 2017). Por ello, también se recurrió a la solución alternativa propuesta por Henseler, Ringle y Sarstedt (citado por Hair Jr., et al., 2017, p.118). Esta consiste en verificar que los intervalos de confianza de los valores HTMT, generados por medio de boostraping, no contengan el valor 1 para cada par de variables latentes del modelo path. Como se puede observar en la Tabla 4, este criterio se cumple en ambos modelos de medida. Lo anterior sugiere que cada par de constructos reflectivos son, empíricamente, distintos.

El cumplimiento de todos los criterios de evaluación de los modelos de medida reflectivos presentados aquí, brindan el soporte suficiente para concluir que las medidas empleadas son confiables y válidas; por ende, apropiadas para realizar los modelos estructurales.

Tabla 4

Intervalos de confianza de los valores HTMT

\begin{tabular}{lll}
\hline Relaciones & $\begin{array}{l}\text { Modelos de medida relativos a construc- } \\
\text { tos reflectivos DEs, CPs, Ac y CIG }\end{array}$ & $\begin{array}{l}\text { Modelos de medida relativos a constructos } \\
\text { reflectivos DEs, CPs, Ac y CII }\end{array}$ \\
\hline Ac $>$ CI & {$[.68 ; .83]$} & {$[.20 ; .48]$} \\
DEs $->$ CI & {$[.64 ; .80]$} & {$[.13 ; .36]$} \\
CPs $->$ CI & {$[.72 ; .85]$} & {$[.21 ; .49]$} \\
CPs $->$ Ac & {$[.90 ; .99]$} & {$[.90 ; .99]$} \\
DEs $->$ Ac & {$[.79 ; .90]$} & {$[.78 ; .90]$} \\
DEs $->$ CPs & {$[.85 ; .94]$} & {$[.84 ; .94]$}
\end{tabular}


Nota: $\mathrm{DEs}=$ Dirección estratégica, $\mathrm{CPs}=$ Capital Psicológico, $\mathrm{Ac}=$ Acuerdo, $\mathrm{CIG}=$ Comportamiento innovador del grupo, $\mathrm{CII}=$ Comportamiento innovador del individuo

Fuente: Elaboración propia

\section{Modelos estructurales}

Modelo estructural referente al comportamiento innovador del grupo

Los valores VIF entre cada grupo de variables latentes predictoras en el modelo estructural se ubicaron entre un mínimo de 1 y un máximo de 3.91; por debajo del umbral de 5 (Hair Jr., et al., 2017, p.194). Lo anterior, sugiere ausencia de problemas de multicolinealidad entre constructos predictores. Tal como lo muestra la Tabla 5, los resultados del modelo estructural muestran coeficientes de trayectoria positivos y estadísticamente significativos. Lo anterior, significa que la direccionalidad estratégica influye de manera positiva sobre el capital psicológico (H2a), lo mismo que en el desarrollo de acuerdo (H4a); a su vez, estos dos últimos constructos impulsan de manera significativa el comportamiento innovador del grupo (H3a y H5a). Además, revela que la direccionalidad estratégica también influye de manera directa sobre el comportamiento innovador del grupo (H1a). Por lo anterior, las hipótesis H1a, H2a, H3a, H4a y H5a logran ser apoyadas.

En general, el modelo apunta a que la direccionalidad estratégica explica un 55.4\% de la varianza (R2) de desarrollo de acuerdo y un 66.4\% de la varianza (R2) del capital psicológico; y por otro lado, que el modelo explica un 55.6\% de la varianza en el comportamiento innovador del grupo (todos estos valores estadísticamente significativos @<.001). En lo relativo a tamaño del efecto, la Tabla 2 muestra que el constructo direccionalidad estratégica tiene un tamaño de efecto grande en las variables latentes desarrollo de acuerdo y capital psicológico, con lo cual, su exclusión en el modelos estructural tiene incidencia importante en su capacidad predictiva; empero, un tamaño de efecto pequeño en el comportamiento innovador del grupo. Por su parte, las variables latentes capital psicológico, y desarrollo de acuerdo tienen un efecto pequeño en el constructo comportamiento innovador del grupo.

Por otro lado, para explorar los posibles mecanismos a través de los cuales la direccionalidad estratégica logra explicar mejor el comportamiento innovador del grupo, se evaluaron los efectos indirectos específicos y los efectos indirectos totales, recurriendo al boostrapping que ofrece la versión 3.0 del Smart-PLS. Como se observa en la Tabla 5 los resultados sugieren que el efecto de la direccionalidad estratégica en el comportamiento innovador del grupo a través del desarrollo de acuerdo resulta ser significativo ( $\mathrm{Q}<.01$ ), lo mismo, el efecto de la direccionalidad estratégica en el comportamiento innovador del grupo mediado por el capital psicológico ( $\mathrm{Q}<.001)$. En este sentido, se lograr corroborar que grupos de trabajo con claridad en objetivos es un elemento alineador que potencia el efecto de ambientes positivos y el 
efecto del logro de acuerdo en los miembros del grupo sobre el comportamiento innovador en los grupos. Lo anterior es constatado por el grado de significancia estadística que aportan los efectos indirectos totales $(\varrho<.001)$.

Tabla5

Coeficientes de trayectoria, nivel de significancia y tamaño del efecto

\begin{tabular}{lllll} 
Trayectoria & Coeficiente & IC 95\% & f2 & IC 95\% \\
\hline Directos & & & & \\
\hline DEs $->$ Ac & $.730 * * *$ & {$[.672 ; .785]$} & $1.143 \mathrm{a}$ & {$[.822 ; 1.160]$} \\
DEs $->$ CPs & $.790 * * *$ & {$[.744 ; .836]$} & $1.662 \mathrm{a}$ & {$[1.237 ; 2.317]$} \\
DEs $->$ CIG & $.169 *$ & {$[.000 ; .339]$} & $.023 \mathrm{c}$ & {$[.000 ; .096]$} \\
CPs $->$ CIG & $.408 * * *$ & {$[.229 ; .585]$} & $.095 \mathrm{c}$ & {$[.029 ; .208]$} \\
Ac $->$ CIG & $.222 * *$ & {$[.073 ; .371]$} & $.035 \mathrm{c}$ & {$[.004 ; .105]$}
\end{tabular}

Indirectos específicos

DEs $->$ Ac $->$ CIG $\quad .162 * * \quad[.053 ; .274]$

DEs $->$ CPs $->$ CIG $\quad .323 * * * \quad[.181 ; .468]$

Indirectos Totales

DEs $->$ CIG $\quad .485 * * * \quad[.361 ; .607]$

Nota: ${ }^{*} \mathrm{Q}<.05 ; * * \mathrm{Q}<.01 ; * * * \mathrm{Q}<.001$

Acorde con Cohen (1998), citado por Hair et al. (2017, p.201): aTamaño del efecto grande (.35), bTamaño del efecto mediano (.15), cTamaño del efecto pequeño (.02). DEs=Dirección estratégica, CPs=Capital Psicológico, $\mathrm{Ac}=$ Acuerdo, $\mathrm{CIG}=$ Comportamiento innovador del grupo, $\mathrm{CII}=$ Comportamiento innovador del individuo.

Fuente: Elaboración propia

\section{Modelo estructural referente al comportamiento innovador del individuo}

Los valores VIF entre cada grupo de variables latentes predictoras en el modelo estructural, se ubicaron entre un mínimo de 1 y un máximo de 3.94; por debajo del umbral de 5 (Hair Jr., et al., 2017, p.194). A diferencia del modelo estructural anterior, los resultados indican que los siguientes efectos directos son los únicos que presentan una relación directa y estadísticamente significativa: la direccionalidad estratégica incide positivamente, tanto en el desarrollo de acuerdo (H4b) como en el capital psicológico (H2b) ( $\varrho<.001)$. Por otro lado, el capital psicológico influye de manera directa y positiva sobre el comportamiento innovador del individuo (H3b). Lo anterior permite dar soporte a las hipótesis H2b, H3b y H4b. Contario a lo hipotetizado, la direccionalidad estratégica presenta una relación inversa con el comportamiento innovador del individuo, pero no significativa, con lo cual, no se da soporte a 
la H1b. Finalmente, el desarrollo de acuerdo presenta un coeficiente de trayectoria positivo, pero no estadísticamente significativo, de esta manera no se logra avalar la hipótesis H5b. Al igual que en el modelo estructural anterior, la direccionalidad estratégica tiene un tamaño del efecto grande en desarrollo de acuerdo y capital psicológico; y pequeño en el comportamiento innovador del individuo. Asimismo, los constructos desarrollo de acuerdo y capital psicológico, muestran un tamaño del efecto pequeño en la variable latente comportamiento innovador del individuo.

Por otro parte, entre los posibles mecanismos a través de los cuales la direccionalidad estratégica logra explicar mejor el comportamiento innovador del individuo, la Tabla 6 sugiere que ninguno de los dos efectos indirectos específicos resulta ser significativo para una mejor explicación del comportamiento innovador del individuo.

Tabla 6

Coeficientes trayectoria, nivel de significancia y tamaño del efecto

\begin{tabular}{|c|c|c|c|c|}
\hline Trayectoria & Coeficientes & IC $95 \%$ & $\mathrm{f} 2$ & IC $95 \%$ \\
\hline \multicolumn{5}{|l|}{ Directos } \\
\hline DEs $->$ Ac & $.731 * * *$ & {$[.673 ; .785]$} & $1.147 \mathrm{a}$ & {$[.826 ; 1.607]$} \\
\hline DEs $->$ CPs & $.790 * * *$ & {$[.742 ; .836]$} & $1.655 \mathrm{a}$ & {$[1.223 ; 2.325]$} \\
\hline DEs $->$ CII & -.109 & {$[-.320 ; .105]$} & $.005 \mathrm{c}$ & {$[.000 ; .041]$} \\
\hline CPs $->$ CII & .263 & {$[.002 ; .525]$} & $.002 \mathrm{c}$ & {$[.000 ; .083]$} \\
\hline Ac $->$ CII & .179 & {$[-.043 ; .397]$} & $.011 \mathrm{c}$ & {$[.000 ; .057]$} \\
\hline \multicolumn{5}{|c|}{ Indirectos específicos } \\
\hline DEs $->\mathrm{Ac}->\mathrm{CII}$ & .131 & {$[-.031 ; .294]$} & & \\
\hline DEs $->$ CPs $->$ CII & $.208^{*}$ & {$[.002 ; .422]$} & & \\
\hline \multicolumn{5}{|l|}{ Indirectos Totales } \\
\hline DEs $->$ CII & $.338 * * *$ & {$[.162 ; .516]$} & & \\
\hline \multicolumn{5}{|c|}{ Nota: $*^{*}<<.05 ; * * \mathrm{Q}<.01 ; * * * \mathrm{Q}<.001$} \\
\hline \multicolumn{5}{|c|}{$\begin{array}{l}\text { Acorde con Cohen (1998), citado por Hair et al. (2017, p.201): aTamaño del efecto grande, bTamaño del efecto } \\
\text { mediano, cTamaño del efecto pequeño. DEs=Dirección estratégica, CPs=Capital Psicológico, Ac=Acuerdo, CI- }\end{array}$} \\
\hline
\end{tabular}

Fuente: Elaboración propia

En las Figuras 2 y 3 se presentan los coeficientes directos de trayectoria de los modelos de medida sobre comportamiento innovador del grupo y comportamiento innovador del individuo, pero, controlando por la variable latente de marcado. Al comparar el tamaño y la significancia de los coeficientes directos de trayectoria de la tabla 5 con los de la figura $2 \mathrm{y}$; de igual 
forma, los de la tabla 6 con los de la figura 3 , se puede corroborar si efectivamente existe un sesgo de varianza común (Rönkkö y Ylitalo, 2011). De acuerdo con estos dos autores, si los coeficientes dejan de ser significativos cuando se controla por la variable de marcado implica la presencia del sesgo, por lo cual, el investigador debería utilizar los resultados controlando por la variable de marcado y realizar una corrección sobre las cargas factoriales estandarizadas. En el caso de los resultados de esta investigación se puede observar claramente que la significancia no cambia; incluso, el tamaño de los coeficientes de trayectoria varía levemente entre los de las tablas 5 y 6 y los de las Figuras 2 y 3 . Este hallazgo sirve de evidencia de que el sesgo de varianza común no afecta los resultados obtenidos.

Figura 2.Modelo estructural de inductores del comportamiento innovador del grupo controlando sesgo varianza común.

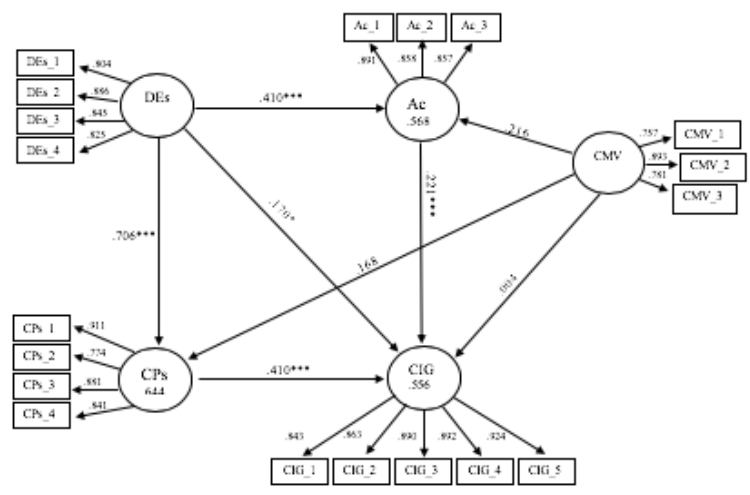

Nota: $*^{\mathrm{Q}<.05 ;} * *{ }^{\mathrm{Q}}<.01 ; * * * \mathrm{Q}<.001$ Des=Dirección estratégica, CPs=Capital Psicológico, Ac=Acuerdo, CIG=Comportamiento innovador del grupo, $\mathrm{CII}=$ Comportamiento innovador del individuo .

Fuente: Elaboración propia 
Figura 3. Modelo estructural de inductores del comportamiento innovador del individuo controlando sesgo varianza común.

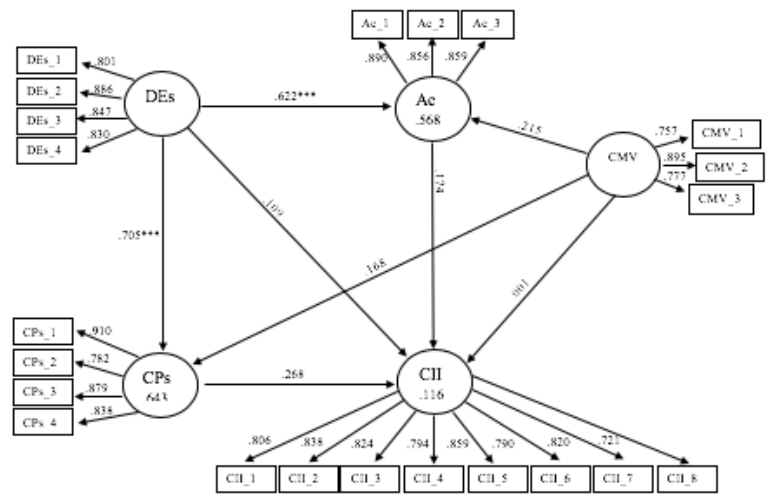

Nota: $* Q<.05 ; * * \mathrm{Q}<.01 ; * * * \mathrm{Q}<.001$. Des=Dirección estratégica, CPs=Capital Psicológico, Ac=Acuerdo, $\mathrm{CIG}=$ Comportamiento innovador del grupo, $\mathrm{CII}=$ Comportamiento innovador del individuo. Fuente: Elaboración propia

\section{Conclusiones}

Con el fin de aportar al conocimiento y discusión sobre factores que influyen el comportamiento innovador del individuo y de los grupos de trabajo, en este estudio se analizó cómo el entramado de relaciones entre la dirección estratégica de los grupos de trabajo, el capital psicológico del grupo y la facilidad para obtener acuerdos en el grupo, inciden sobre esos dos tipos de comportamiento innovador.

Los resultados obtenidos permiten avalar la hipótesis de que la direccionalidad estratégica influye de forma positiva el comportamiento innovador del grupo (H1a). Este resultado está acorde con lo señalado por otros investigadores acerca de cómo los grupos de trabajo con objetivos claramente definidos y una estrategia clara de cara al futuro son más propensos a desarrollar nuevos métodos de trabajo (Somech y Drach-Zahavy, 2011), lo mismo que, mostrar una orientación innovadora (Amabile, 1997). En esta búsqueda por alcanzar los objetivos pueden surgir ideas novedosas (Shoham et al., 2012), se logre disponer de un enfoque y direccionamiento para discernir en lo que se debe innovar (Somech y Drach-Zahavy, 2011), de flexibilidad para tomar los riesgos que trae consigo la innovación (Moon, 1999), y de planificación para llevar a cabo proyectos o ideas (Koch y Hauknes, 2005).

El estudio confirma que la direccionalidad estratégica también influye el comportamiento innovador del grupo de forma indirecta a través del capital psicológico (H2a) y el acuerdo (H3a), ya que se observa que la direccionalidad estratégica influye directamente el capital 
psicológico (H2a) y el acuerdo (H4a), y estos constructos a su vez muestran una influencia directa sobre el comportamiento innovador del grupo (H3a y H5a). El contar con una direccionalidad clara acerca de lo que se quiere alcanzar y cómo lograrlo puede ser un medio que facilite el alcance de acuerdos para crear propósitos y sinergias comunes (Gilson y Shalley, 2004). A su vez, los acuerdos y consensos son necesarios para el desarrollo de la innovación, ya que generalmente la implementación de una idea creativa requiere de la participación de diferentes individuos (Caldwell y O'Reilly III, 2003; de Faria, y de Alencar, 1996). De esta manera, si en los grupos se dificulta alcanzar consensos es posible que se mitigue el comportamiento innovador colectivo.

Por otra parte, poseer una direccionalidad clara podría fomentar actitudes positivas en el grupo de trabajo (Luthans et al., 2008), lo cual explica la relación significativa y directa que existe entre direccionalidad estratégica y capital psicológico colectivo. A su vez el capital psicológico colectivo tiene una fuerte asociación con el comportamiento innovador del grupo. Se ha demostrado que el optimismo se asocia con el cambio, la toma de riesgos y cumplimiento de objetivos (Green et al., 2004), y permite adaptarse a situaciones adversas o retos (Walumbwa et al., 2011); como los que plantea una innovación. Por lo cual, en un grupo con mayor capital psicológico puede existir mayor disposición de generar e implementar ideas novedosas.

En lo referente al comportamiento innovador del individuo se evidenció la hipótesis de relación directa entre capital psicológico del grupo y comportamiento innovador del individuo (H3b), en contraste con direccionalidad estratégica y acuerdo (H1b y H5b). Estos hallazgos sugieren que tanto el acuerdo como la direccionalidad estratégica influyen el comportamiento y las decisiones colectivas en relación con impulsar la innovación, pero no así el comportamiento y las decisiones individuales. Una posible explicación de estos resultados es que los ítems de comportamiento innovador del grupo están más enfocados a los esfuerzos para implementar ideas innovadoras, para lo cual se puede requerir de acuerdos de grupo y una direccionalidad estratégica que permitan respaldar y legitimar la necesidad de llevar adelante una idea innovadora, así como el trabajo y planificación de grupo que se necesita para su implementación. Por otra parte, los ítems de comportamiento innovador del individuo no están tan enfocados en la implementación, sino más bien en la iniciativa del sujeto por buscar nuevas ideas y ser creativo, lo cual, puede estar más asociado a actitudes y rasgos propios del individuo que a elementos relacionados con la direccionalidad estratégica del grupo o la facilidad de alcanzar acuerdos.

Otra posible explicación por la cual no se encuentra relación directa entre acuerdo y direccionalidad estratégica con comportamiento innovador del individuo, podría estar relacionada al hecho de que el propio individuo responde por su comportamiento innovador, y quizás por el sesgo de la deseabilidad social sus respuestas estén sobreestima- 
das. Sin embargo, esta es una posibilidad que no se puede aseverar a partir de los datos recopilados en esta investigación.

\section{Implicaciones para la gestión pública}

Los resultados del estudio sugieren que un factor trascendental para impulsar el comportamiento innovador de los grupos de trabajo dentro de las organizaciones es el establecimiento de una dirección clara acerca de qué es lo que se quiere alcanzar como grupo, así como las estrategias para lograr los objetivos a través de una continua planificación. Estos elementos no solo afectarían de manera directa el comportamiento innovador del grupo, al dar direccionalidad sobre los que se debe innovar, sino también de forma indirecta, generando facilidades para alcanzar los acuerdos que se requieren para innovar y un capital psicológico positivo que le permite al grupo enfrentar las dificultades que surgen cuando se buscan realizar cambios.

A su vez, los resultados sugieren que, para impulsar el comportamiento innovador de los individuos y grupos, los equipos deben enfocarse en crear un ambiente optimista y positivo, en el que se pueden alcanzar las metas y salir adelante ante cualquier dificultad. En este sentido, el hecho de que las capacidades psicológicas sean medibles, desarrollables y gestionables (Luthans y Youssef, 2004), despierta el interés por el desarrollo de programas de intervención con encargados de recursos humanos (e.g.: capacitación) (Luthans et al., 2006).

\section{Limitaciones y futuras líneas de investigación}

Una limitación metodológica en la investigación ha sido el uso de la misma fuente de información para medir el comportamiento innovador del individuo y del grupo, de igual modo, para los restantes constructos. Esto puede ocasionar que el sesgo de varianza común infle los coeficientes de trayectoria (Podsakoff et al., 2012). Para mitigar este sesgo se utilizó una escala de medida distinta para los constructos de comportamiento innovador, además se trató de controlar el efecto del sesgo agregando una variable de marcado en el análisis del modelo PLS (Podsakoff et al., 2012). A pesar del uso de estos métodos de prevención, sugeridos en la literatura, cabe decir que el mecanismo ideal para prevenir dicho sesgo es utilizar una fuente de información distinta para los constructos de comportamiento innovador y otra distinta para los restantes constructos (Podsakoff et al., 2012). Además, hubiese sido ideal medir el comportamiento innovador del grupo a partir de un agregado de las respuestas de los individuos que lo componen, para ello habría que buscar los diferentes grupos de trabajo para aplicar las entrevistas entre sus integrantes.

Otro elemento para señalar es que, no obstante, tanto el método de Fornell y Larcker como los intervalos de confianza de los valores HTMT sugieren que existe validez discriminante, los criterios se cumplieron cerca del límite entre los constructos capital psicológico, dirección 
estratégica y acuerdo. Por ello, será recomendable agregar ítems adicionales que permiten establecer una discriminación más marcada.

El estudio se llevó a cabo con funcionarios públicos, por lo cual, una oportunidad de investigación futura será comprobar estas relaciones en el ámbito privado y en otros contextos culturales, mejorando las limitantes metodológicas señaladas, así como estudiar no solo el comportamiento innovador sino también la implementación de innovaciones.

En esta investigación queda pendiente una cuestión futura por responder, a saber, por qué motivo la direccionalidad estratégica y el acuerdo no presentan relación directa con el comportamiento innovador del individuo, en cambio sí mostró relación significativa con el comportamiento innovador del grupo. Si bien es cierto, se han conjeturado dos posibles motivos en párrafos previos (una, con la forma de medir el comportamiento innovador y la otra con una posible limitante relacionada con la unidad informante); un futuro estudio podría tratar de buscar respuestas alternativas a esta inquietud. Además, explorar otros constructos con potencial de influir sobre el comportamiento innovador de grupo y no así del individuo.

Por último, Anderson, Potočnik y Zhou (2014) mencionan la carencia de estudios que aborden esta temática de innovación desde enfoque multinivel. En otras palabras, que analicen el efecto de variables de los niveles organizativo, de grupos y de individuos sobre el comportamiento innovador y la generación de innovación en grupos e individuos. En consecuencia, desde dicho enfoque, conocer cómo las variables, en sus diferentes niveles influyen sobre el comportamiento innovador, lo mismo que, la interacción que existe entre ellas se plantea como un reto de abordaje a futuro.

\section{Referencias}

Abbas, M., y Raja, U. (2015). Impact of psychological capital on innovative performance and job stress. Canadian Journal of Administrative Sciences/Revue Canadienne des Sciences de l'Administration, 32(2), 128-138. https:// doi.org/10.1002/cjas.1314

Amabile, T. M. (1997). Motivating creativity in organizations: On doing what you love and loving what you do. California Management Review, 40(1), 39-58. https://doi.org/10.2307/41165921

Anderson, N. y West, M. A. (1996). The Team Climate Inventory: Development of the TCI and its applications in teambuilding for innovativeness. European Journal of Work and Organizational Psychology, 5(1), 53-66. https:// doi.org/10.1080/13594329608414840

Anderson, N., y West, M. A. (1998). Measuring climate for work group innovation: development and validation of the team climate inventory. Journal of Organizational Behavior, 19, 235-258.

https://doi.org/10.1002/(sici)1099-1379(199805)19:3<235::aid-job837>3.0.co;2-c

Anderson, N., Potočnik, K., y Zhou, J. (2014). Innovation and creativity in organizations: A state-of-the-science review, prospective commentary, and guiding framework. Journal of Management, 40(5), 1297-1333. https:// doi.org/10.1177/0149206314527128

Avey, J. B., Wernsing, T. S., y Luthans, F. (2008). Can positive employees help positive organizational change? Impact of psychological capital and emotions on relevant attitudes and behaviors. The Journal of Applied Behavioral Science, 44(1), 48-70.

https://doi.org/10.1177/0021886307311470 
Bandura, A. (1997). Self-efficacy: The exercise of control. New York: Freeman. https://doi.org/10.1037/e622652007-001

Bart, C. K., Bontis, N., y Taggar, S. (2001). A model of the impact of mission statements on firm performance. Management Decision, 39(1), 19-35. https://doi.org/10.1108/eum0000000005404

Beaton, D., y Guillemin, F. (2000). Guidelines for the process of cross-cultural adaptation of self- report measures. SPINE, 25(24), 3186-3191.

https://doi.org/10.1097/00007632-200012150-00014

Beatty, P. C., y Willis, G. B. (2007). Research synthesis: The practice of cognitive interviewing. Public Opinion Quarterly, 71(2), 287-311. https://doi.org/10.1093/poq/nfm006

Boswell, W. (2006). Aligning employees with the organization's strategic objectives: Out of 'line of sight', out of mind. The International Journal of Human Resource Management, 17(9), 1489-1511. https://doi. org/10.1080/09585190600878071

Bowen, D. E., y Ostroff, C. (2004). Understanding HRM-firm performance linkages: The role of the "strength" of the HRM system. Academy of Management Review, 29(2), 203-221. https://doi.org/10.2307/20159029

Brislin, R. (1986). The Wording and translation of research instruments. En W.J. Lonner, y J. W. Berry (Ed.), Field methods in cross-cultural research (p.137-164). California, USA: Sage Publications. https://doi.org/10.2307/2349027

Caldwell, D. F., y O'Reilly III, C. A. (2003). The determinants of team-based innovation in organizations: The role of social influence. Small Group Research, 34(4), 497-517.

https://doi.org/10.1177/1046496403254395

Camelo-Ordaz, C., Fernández-Alles, M. D. L. L., \& Valle-Cabrera, R. (2008). Top management team's vision and human resources management practices in innovative Spanish companies. The International Journal of Human Resource Management, 19(4), 620-638. https://doi.org/10.1080/09585190801953665

Cameron, K., Mora, C., Leutscher, T., y Calarco, M. (2011). Effects of positive practices on organizational effectiveness. The Journal of Applied Behavioral Science, 47(3), 266-308. https://doi.org/10.1177/0021886310395514

Cardinal,L. B. (2001). Technological innovation in the pharmaceutical industry: The use of organizational control in managing research and development. Organization Science, 12(1), p. 19-36. https://doi.org/10.1287/orsc.12.1.19.10119

Chin, W. W. (1998). The partial least squares approach to structural equation modeling. Modern Methods for Business Research, 295(2), 295-336.

Colton, D., y Covert, R.W. (2007). Designing and constructing instruments for social research and evaluation. California, USA: John Wiley \& Sons, Inc.

https://doi.org/10.1177/1035719x0800800213

de Faria, M. D. F. B., y de Alencar, E. M. L.S. (1996). Estímulos e barreiras à criatividade no ambiente de trabalho. Revista de Administracão, 31(2), 50-61.

https://doi.org/10.1590/s0103-166x2010000400004

De Jong, J., y Den Hartog, D. (2010). Measuring innovative work behaviour. Creativity and Innovation Management, 19(1), 23-36.

https://doi.org/10.1111/j.1467-8691.2010.00547.x

Denison, D.R., Janovics, J., y Young, J. (2006). Diagnosing Organizational Cultures: Validating a Model and Method. Documento de trabajo. Denison Consulting Group.

Dereli, D. D. (2015). Innovation management in global competition and competitive advantage. Procedia-Social and Behavioral Sciences, 195, 1365-1370. https://doi.org/10.1016/j.sbspro.2015.06.323

DeVellis, R.F. (2012). Scale development: Theory and applications (Tercera edición). California, USA: Sage Publications Ltd.

Fey, C. F., y Denison, D. R. (2003). Organizational culture and effectiveness: can American theory be applied in Russia?. Organization Science, 14(6), 686-706.

https://doi.org/10.2139/ssrn.577141

Franz, N. (2004). Self-Directed Work Teams: The Antidote for "Heroic Suicide.”. Journal of Extension, 42(2), disponible en: https://works.bepress.com/nancy_franz/6/download/ y consultado: 25/01/2017 
Gilson, L. L.,y Shalley, C. E. (2004). A little creativity goes a long way: An examination of teams' engagement in creative processes. Journal of Management, 30(4), 453-470.

https://doi.org/10.1016/j.jm.2003.07.001

Green Jr, K. W., Medlin, B., y Whitten, D. (2004). Developing optimism to improve performance: an approach for the manufacturing sector. Industrial Management \& Data Systems, 104(2), 106-114. https://doi. org/10.1108/02635570410522071

Gully, S. M., Incalcaterra, K. A., Joshi, A., y Beaubien, J. M. (2002). A meta-analysis of team-efficacy, potency, and performance: interdependence and level of analysis as moderators of observed relationships. Journal of Applied Psychology, 87(5), 819-832.

https://doi.org/10.1037/0021-9010.87.5.819

Hair Jr., J., Hult, G., Ringle, C., y Sarstedt, M. (2017). A primer on partial least squares structural equation modeling (PLS-SEM) (Segunda edición). California, USA: Sage Publications.

Henseler, J., Ringle, C. M., y Sinkovics, R. R. (2009). The use of partial least squares path modeling in international marketing. En R. Sinkovics, P.N. Ghauri (Eds.). New challenges to international marketing, (advances in international marketing, 20) (p.277-319). Bingley: Emerald Group Publishing Limited.

Hogan, S. J., y Coote, L. V. (2014). Organizational culture, innovation, and performance: A test of Schein's model. Journal of Business Research, 67(8), 1609-1621. https://doi.org/10.1016/j.jbusres.2013.09.007

Högl, M., y Parboteeah, K. P. (2003). Goal setting and team performance in innovative projects: On the moderating role of teamwork quality. Small Group Research, 34(1), 3-19.

https://doi.org/10.1177/1046496402239575

Holland, S., Gaston, K., y Gomes, J. (2000). Critical success factors for cross $\square$ functional teamwork in new product development. International Journal of Management Reviews, 2(3), 231-259. https://doi.org/10.1111/1468-2370.00040

Holleman, G., Poot, E., Mintjes-de Groot, J., y van Achterberg, T. (2009). The relevance of team characteristics and team directed strategies in the implementation of nursing innovations: a literature review. International Journal of Nursing Studies, 46(9), 1256-1264. https://doi.org/10.1016/j.ijnurstu.2009.01.005

Hülsheger, U. R., Anderson, N., y Salgado, J. F. (2009). Team-level predictors of innovation at work: a comprehensive meta-analysis spanning three decades of research. Journal of Applied Psychology, 94(5),1128-1145. https://doi.org/10.1037/a0015978

Jafri, H. (2012). Psychological capital and innovative behaviour: An empirical study on apparel fashion industry. Journal of Contemporary Management Research, 6(1), 42-52.

Jamrog, J., Vickers, M., y Bear, D. (2006). Building and sustaining a culture that supports innovation. People and Strategy, 29(3), 9-19.

Janssen, O. (2000). Job demands, perceptions of effort $\square$ reward fairness and innovative work behavior. Journal of Occupational and Organizational Psychology, 73(3), 287-302.

https://doi.org/10.1348/096317900167038

Janssen, O., Van de Vliert, E., y West, M. (2004). The bright and dark sides of individual and group innovation: A special issue introduction. Journal of Organizational Behavior, 25(2), 129-145. https://doi.org/10.1002/job.242

Jassawalla, A. R., y Sashittal, H. C. (2002). Cultures that support product-innovation processes. The Academy of Management Executive, 16(3), 42-54. https://doi.org/10.5465/ame.2002.8540307

Jing, F. F., Avery, G. C., y Bergsteiner, H. (2014). Enhancing performance in small professional firms through vision communication and sharing. Asia Pacific Journal of Management, 31(2), 599-620. https://doi.org/10.1007/ s10490-013-9345-9

Katzenbach, J. R., y Smith, D. K. (1993). The discipline of teams. Harvard Business Review, 71(2), 111-120.

Kleysen, R. F y Street, C. T. (2001). Toward a multi-dimensional measure of individual innovative behavior. Journal of Intellectual Capital, 2(3), 284-296.

https://doi.org/10.1108/eum0000000005660

Koch, P., y Hauknes, J. (2005). On Innovation in the Public Sector. Publin Report No. D20, NIFU STEP, Oslo. 
Kozlowski, S. W., y Ilgen, D. R. (2006). Enhancing the effectiveness of work groups and teams. Psychological Science in the Public Interest, 7(3), 77-124. https://doi.org/10.1177/1745691617697078

Krause, D. E. (2004). Influence-based leadership as a determinant of the inclination to innovate and of innovation-related behaviors: An empirical investigation. The leadership quarterly, 15(1), 79-102. https://doi.org/10.1016/j. leaqua.2003.12.006

Latham, G. P., y Yukl, G. A. (1975). A review of research on the application of goal setting in organizations. Academy of Management Journal, 18(4), 824-845. https://doi.org/10.5465/255381

Levin, I. M. (2000). Vision revisited: Telling the story of the future. The Journal of Applied Behavioral Science, 36(1), 91-107. https://doi.org/10.1177/0021886300361005

Locke, E. A. (1996). Motivation through conscious goal setting. Applied and Preventive Psychology, 5(2), 117-124. https://doi.org/10.1016/s0962-1849(96)80005-9

Locke, E. A., Shaw, K. N., Saari, L. M., y Latham, G. P. (1981). Goal setting and task performance: 1969-1980. Psychological Bulletin, 90(1), 125-152.

https://doi.org/10.21236/ada086584

Locke, E. A., y Latham, G. P. (2002). Building a practically useful theory of goal setting and task motivation: A 35-year odyssey. American Psychologist, 57(9), 705-717.

https://doi.org/10.1037/0003-066x.57.9.705

Luke, B., Verreynne, M. L., y Kearins, K. (2010). Innovative and entrepreneurial activity in the public sector: The changing face of public sector institutions. Innovation, 12(2), 38-153. https://doi.org/10.5172/impp.12.2.138

Luthans, F., Avey, J. B., y Patera, J. L. (2008). Experimental analysis of a web-based training intervention to develop positive psychological capital. Academy of Management Learning \& Education, 7(2), 209-221.

https://doi.org/10.5465/amle.2008.32712618

Luthans, F., Avey, J., Avolio, B., Norman, S.M., y Combs, G. (2006). Psychological Capital Development: Toward a Micro-Intervention. Management Department Faculty Publications. 146, Universidad de Nebraska. Disponible en: https://digitalcommons.unl.edu/cgi/viewcontent.cgi?article=1152\&context=managementfacpub, consultado 05/02/2017

Luthans, F., Avolio, B. J., Avey, J. B., y Norman, S. M. (2007). Positive psychological capital: Measurement and relationship with performance and satisfaction. Personnel Psychology, 60(3), 541-572. https://doi.org/10.1111/ j.1744-6570.2007.00083.x

Luthans, F., Avolio, B. J., Walumbwa, F. O., y Li, W. (2005). The psychological capital of Chinese workers: Exploring the relationship with performance. Management and Organization Review, 1(2), 249-271.

https://doi.org/10.1111/j.1740-8784.2005.00011.x

Luthans, F., y Youssef, C. M. (2004). Human, social, and now positive psychological capital management: Investing in people for competitive advantage. Organizational Dynamics, 33(2), 143-160. https://doi.org/10.1016/j. orgdyn.2004.01.003

Luthans, F., Youssef, C. M., y Avolio, B. J. (2007). Psychological capital: Developing the human competitive edge. Oxford, UK: Oxford University Press.

Messmann, G., y Mulder, R. H. (2012). Development of a measurement instrument for innovative work behaviour as a dynamic and context-bound construct. Human Resource Development International, 15(1), 43-59. https:// doi.org/10.1080/13678868.2011.646894

Moon, M. J. (1999). The pursuit of managerial entrepreneurship: does organization matter? Public Administration Review, 15(1), 31-43. https://doi.org/10.2307/977477

Norton, R.S., y Kaplan, D.P. (2004) Mapas estratégicos: Convirtiendo los activos intangibles en resultados tangibles. Barcelona, España: Gestión 2000.

O'Leary-Kelly, A. M., Martocchio, J. J., y Frink, D. D. (1994). A review of the influence of group goals on group performance. Academy of Management Journal, 37(5), 1285-1301. https://doi.org/10.2307/256673

Parzefall, M. R., Seeck, H., y Leppänen, A. (2008). Employee innovativeness in organizations: a review of the antecedents. Finnish Journal of Business Economics, 20(8), 165-182. 
Pearce, C. L., y Ensley, M. D. (2004). A reciprocal and longitudinal investigation of the innovation process: The central role of shared vision in product and process innovation teams (PPITs). Journal of Organizational Behavior, 25(2), p. 259-278. https://doi.org/10.1002/job.235

Podsakoff, P. M., MacKenzie, S. B., y Podsakoff, N. P. (2012). Sources of method bias in social science research and recommendations on how to control it. Annual Review of Psychology, 63, 539-569. https://doi.org/10.1146/ annurev-psych-120710-100452

Potts, J., y Kastelle, T. (2010). Public sector innovation research: What's next?. Innovation, 12(2), 122-137. https:// doi.org/10.5172/impp.12.2.122

Porter, M. E. (1991). Towards a dynamic theory of strategy. Strategic Management Journal, 12, 95-117. https://doi. org/10.1002/smj.4250121008

Post, C. (2012). Deep-level team composition and innovation: The mediating roles of psychological safety and cooperative learning. Group \& Organization Management, 37(5), 555-588. https://doi.org/10.1177/1059601112456289

Ratnaningsih, I. Z., Prasetyo, A. R., y Prihatsanti, U. (2016). Predicting innovative behavior among employees in a manufacturing company: the role of psychological capital.Anima, 31(2), 84-90. https://doi.org/10.24123/aipj.v31i2.567

Rego, A., Sousa, F., Marques, C., y Cunha, M. P. (2012). Authentic leadership promoting employees' psychological capital and creativity. Journal of Business Research, 65(3), 429-437. https://doi.org/10.1016/j.jbusres.2011.10.003

Revilla, E., y Rodríguez, B. (2011). Team vision in product development: How knowledge strategy matters. Technovation, 31(2-3), 118-127. https://doi.org/10.1016/j.technovation.2010.10.007

Ringle, C., Wende, S., y Becker, J. (2015). http://www.smartpls.com. Consultado 20/05/2017

Romero, I., y Martínez-Román, J. A. (2012). Self-employment and innovation. Exploring the determinants of innovative behavior in small businesses. Research Policy, 41(1), 178-189. https://doi.org/10.1016/j.respol.2011.07.005

Rönkkö, M., y Ylitalo, J. (2011). PLS marker variable approach to diagnosing and controlling for method variance. En Proceedings of the International Conference on Information (ICIS 2011). Paper 8. Shanghai. Disponible en: http://citeseerx.ist.psu.edu/viewdoc/download?doi=10.1.1.847.3238\&rep=rep1\&type=pdf, consultado: 20/05/2017

Shalley, C. E., y Gilson, L. L. (2004). What leaders need to know: A review of social and contextual factors that can foster or hinder creativity. The Leadership Quarterly, 15(1), 33-53. https://doi.org/10.1016/j.leaqua.2003.12.004

Schneider, B. (1987). The people make the place. Personnel Psychology, 40(3), 437-453. https://doi. org/10.4324/9780203809549

Schuckert, M., Kim, T. T., Paek, S., y Lee, G. (2018). Motivate to innovate: How authentic and transformational leaders influence employees' psychological capital and service innovation behavior. International Journal of Contemporary Hospitality Management, 30(2), 776-796. https://doi.org/10.1108/ijchm-05-2016-0282

Scott, S. G., y Bruce, R. A. (1994). Determinants of innovative behavior: A path model of individual innovation in the workplace. Academy of management journal, 37(3), 580-607. https://doi.org/10.5465/256701

Shani, N., y Divyapriya, P. (2011). A Role of Innovative Idea Management in HRM. International Journal of Management, 2(1), 69-78.

Shoham, A., Vigoda-Gadot, E., Ruvio, A., y Schwabsky, N. (2012). Testing an organizational innovativeness integrative model across cultures. Journal of Engineering and Technology Management, 29(2), 226-240. https:// doi.org/10.1016/j.jengtecman.2012.01.002

Smith-Castro, V., y Molina, M. (2011). La entrevista cognitiva: Guía para su aplicación en la evaluación y mejoramiento de instrumentos de papel y lápiz. Serie Cuadernos Metodológicos. San José, CR.: Instituto de Investigaciones Psicológicas, Universidad de Costa Rica.

Somech,A., y Drach-Zahavy, A. (2011). Coping with work-family conflict from a cross cultural perspective. Ponencia presentada en biennial meeting for the International Association of Cross-Cultural Psychology, Estambul, Turquía.

Sweetman, D., Luthans, F., Avey, J. B., y Luthans, B. C. (2011). Relationship between positive psychological capital and creative performance. Canadian Journal of Administrative Sciences/Revue Canadienne des Sciences de l'Administration, 28(1), 4-13. https://doi.org/10.1002/cjas.175

Van de Ven, A. H. (1986). Central problems in the management of innovation. Management Science, 32(5), 590-607. https://doi.org/10.1287/mnsc.32.5.590 
Van Rijnsoever, F. J., Meeus, M. T., y Donders, A. R. T. (2012). The effects of economic status and recent experience on innovative behavior under environmental variability: An experimental approach. Research Policy, 41(5), 833-847. https://doi.org/10.1016/j.respol.2012.02.005

Vancouver, J. B., Millsap, R. E., y Peters, P. A. (1994). Multilevel analysis of organizational goal congruence. Journal of Applied Psychology, 79(5), 666-679. https://doi.org/10.1037/0021-9010.79.5.666

Vargas-Halabí, T. y Mora-Esquivel, R. (2017). Adaptación y dimensionalidad de la escala DOCS en el contexto organizacional costarricense: aplicación de la entrevista cognitiva y el análisis factorial confirmatorio. Revista Costarricense de Psicología, 36(2), 199-226. https://doi.org/10.22544/rcps.v36i02.06

Vargas-Halabí, T., Mora-Esquivel, R., y Siles, B. (2017). Intrapreneurial competencies: development and validation of a measurement scale. European Journal of Management and Business Economics, 26(1), 86-111. https://doi. org/10.1108/ejmbe-07-2017-006

Walumbwa, F. O., Luthans, F., Avey, J. B., y Oke,A. (2011). Retracted: Authentically leading groups: The mediating role of collective psychological capital and trust. Journal of Organizational Behavior, 32(1), 4-24. https://doi. org/10.1002/job.653

Wojtczuk-Turek, A., y Turek, D. (2015). Innovative behaviour in the workplace: The role of HR flexibility, individual flexibility and psychological capital: the case of Poland. European Journal of Innovation Management, 18(3), 397-419.

https://doi.org/10.1108/ejim-03-2014-0027

Yuan, F., y Woodman, R. W. (2010). Innovative behavior in the workplace: The role of performance and image outcome expectations. Academy of Management Journal, 53(2), 323-342. https://doi.org/10.1108/dlo.2010.08124fad.010

Zubair, A., y Kamal, A. (2015). Work related flow, psychological capital, and creativity among employees of software houses. Psychological Studies, 60(3), 321-331. https://doi.org/10.1007/s12646-015-0330-x 
Tabla A1

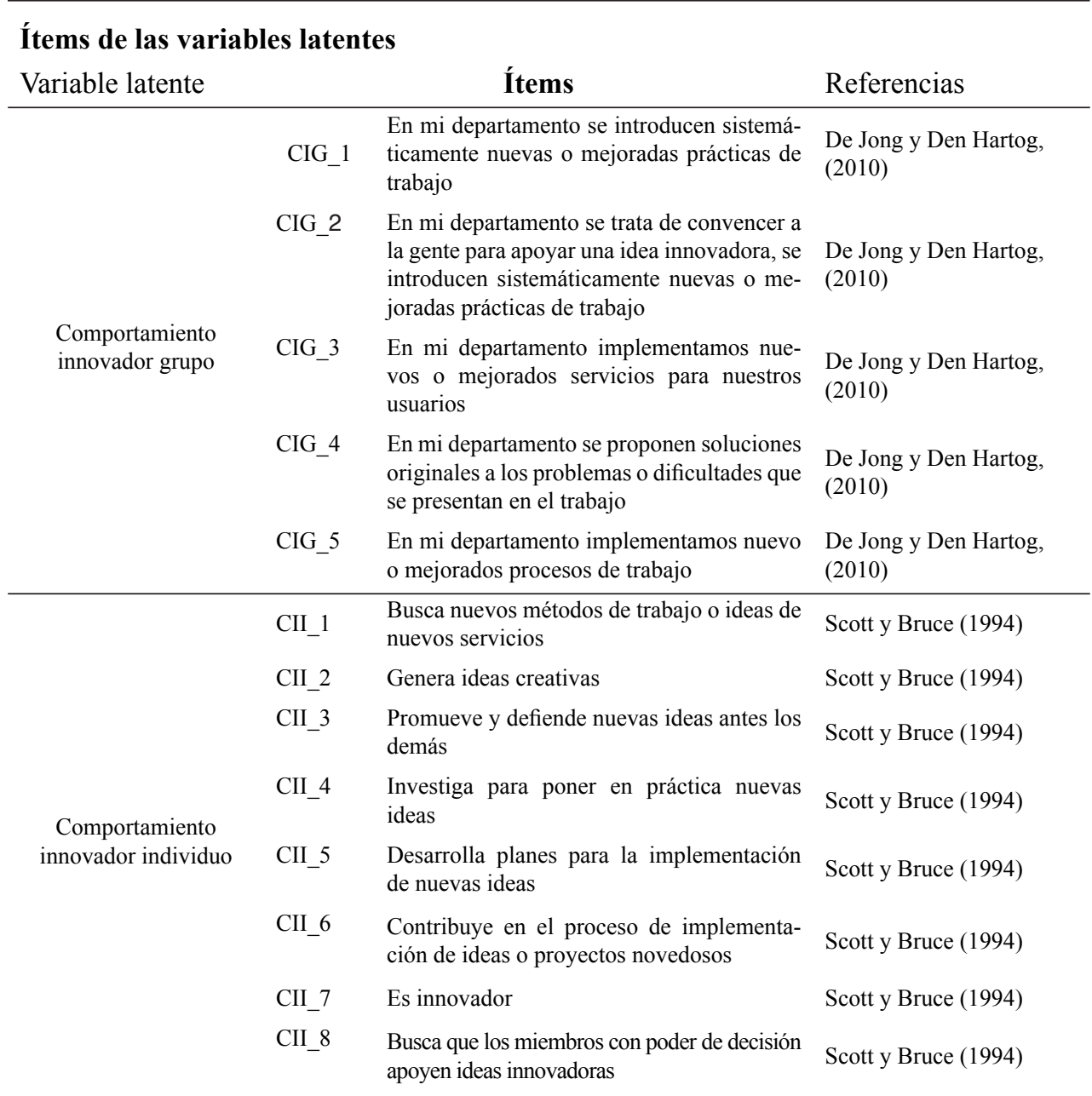




\begin{tabular}{|c|c|c|c|}
\hline \multirow{4}{*}{$\begin{array}{l}\text { Direccionalidad es- } \\
\text { tratégica }\end{array}$} & DEs_1 & $\begin{array}{l}\text { En mi departamento comparamos continua- } \\
\text { mente nuestro progreso con los objetivos } \\
\text { fijados }\end{array}$ & Denison et al. (2006) \\
\hline & DEs_2 & $\begin{array}{l}\text { Este departamento tiene estrategias claras } \\
\text { para cumplir sus metas }\end{array}$ & Denison et al. (2006) \\
\hline & DEs_3 & $\begin{array}{l}\text { Este departamento tiene una misión clara } \\
\text { que le otorga sentido y rumbo a nuestro } \\
\text { trabajo }\end{array}$ & Denison et al. (2006) \\
\hline & DEs_4 & $\begin{array}{l}\text { Mi departamento se caracteriza por una } \\
\text { planificación constante del trabajo que ha- } \\
\text { cemos }\end{array}$ & Original \\
\hline \multirow{4}{*}{ Capital Psicológico } & CPs_1 & $\begin{array}{l}\text { En mi departamento hay optimismo de los } \\
\text { resultados que obtendremos a futuro con el } \\
\text { trabajo que realizamos }\end{array}$ & Walumbwa, et al. (2011) \\
\hline & CPs_2 & $\begin{array}{l}\text { En mi departamento hay una sensación de } \\
\text { seguridad de que se alcanzará el éxito en las } \\
\text { tareas }\end{array}$ & Walumbwa, et al. (2011) \\
\hline & CPs_3 & $\begin{array}{l}\text { Cuando enfrentamos dificultades propias de } \\
\text { nuestras tareas somos muy insistentes en sa- } \\
\text { car adelante lo que nos proponemos }\end{array}$ & Original \\
\hline & CPs_4 & $\begin{array}{l}\text { En mi departamento siempre se ve el lado } \\
\text { bueno de las cosas con respecto a nuestro } \\
\text { trabajo }\end{array}$ & Walumbwa, et al. (2011) \\
\hline \multirow{3}{*}{ Acuerdo } & Ac_1 & $\begin{array}{l}\text { Cuando existe desacuerdos trabajamos in- } \\
\text { tensamente para encontrar soluciones donde } \\
\text { todos ganen }\end{array}$ & Denison et al. (2006) \\
\hline & Ac_2 & $\begin{array}{l}\text { Generalmente las personas tienen disposi- } \\
\text { ción de ceder su interés particular para lo- } \\
\text { grar acuerdos en el grupo }\end{array}$ & Original \\
\hline & Ac_3 & $\begin{array}{l}\text { Nos resulta fácil lograr consenso aún en te- } \\
\text { mas difíciles }\end{array}$ & Denison et al. (2006) \\
\hline
\end{tabular}

